\title{
HYPERGEODESIC MAPPING OF A SURFACE ON A PLANE*
}

\author{
BY \\ ERNEST P. LANE
}

\section{INTRODUCTION}

The purpose of this paper is to study a projective analogue of the metric problem $†$ of Beltrami, to map a surface of ordinary space on a plane so that the geodesics on the surface correspond to the straight lines in the plane. A two-parameter family of hypergeodesics on a surface in ordinary space is defined $\ddagger$ by a differential equation of the same form as the equation of the geodesics, in which, however, the coefficients are arbitrary given functions of the parameters on the surface instead of being the well known formulas containing the Christoffel symbols that appear in the familiar equation of the geodesics. The problem under consideration, then, is to set up a one-toone correspondence between the points of the surface and the points of a plane so that a given covariant family of hypergeodesics shall correspond to the straight lines of the plane.

In the metric theory it is customary not only to suppose that two corresponding points on the surface and in the plane have the same curvilinear coördinates, but also that these are the abscissa and ordinate of the point in the plane. In the projective theory here presented it is found to be convenient first to normalize the curvilinear coördinates on the surface according to Fubini, and then to suppose that the curvilinear coördinates in the plane are the same as on the surface, so that Wilczynski's theory of plane nets is employed in studying the correspondence.

In §2, the theory of Fubini's canonical form of the differential equations of a surface is briefly summarized in so far as it will be needed in this paper. In $\S 3$, the theory of hypergeodesics, including three examples, is similarly treated; and likewise in $\$ 4$, Wilczynski's theory of plane nets.

The theory of hypergeodesic mapping is constructed in $\$ 5$. Three examples of this mapping are discussed in §6. Green's congruentially asso-

* Presented to the Society, April 18, 1930; received by the editors January 25, 1930.

$\dagger$ Beltrami, Annali di Matematica, (1), vol. 7 (1865), pp. 185-204; or else, Opere Complete, vol. 1, pp. 262-280. See also Bianchi, Lezioni, 3d edition, vol. 1, p. 310. For other extensions of the problem of Beltrami, see Kasner, The generalized Beltrami problem concerning geodesic representation, these Transactions, vol. 4 (1903), pp. 149-152.

$\ddagger$ Fubini and Cech, Geometria Proiettiva Differenziale, vol. 1, p. 133. Wilczynski, Some generalizations of geodesics, these Transactions, vol. 23 (1922), p. 283. 
ciated net is connected with the theory in $\$ 7$. Finally, another method of mapping a surface on a plane is suggested in $\$ 8$.

\section{ANALYTIC DEFINITION OF A SURFACE}

A surface in ordinary space can be defined, except possibly for a projective transformation, by a pair of linear homogeneous partial differential equations of the second order, whose coefficients satisfy certain integrability conditions. In this section we shall recapitulate as much as will be needed of the well known theory of the analytic representation of a surface by a pair of equations in Fubini's canonical form.

Let the parametric equations, in projective homogeneous coördinates, of a non-ruled surface $S$ in ordinary space, be

$$
x^{(i)}=x^{(i)}(u, v) \quad(i=1, \cdots, 4) .
$$

If the asymptotic curves on the surface $S$ are parametric and if the proportionality factor of the homogeneous coördinates is suitably chosen, the coördinates $x$ satisfy a system of two equations in Fubini's canonical form,

$$
\begin{array}{ll}
x_{u u} & =p x+\theta_{u} x_{u}+\beta x_{v}, \\
x_{v v} & =q x+\gamma x_{u}+\theta_{v} x_{v}
\end{array} \quad(\theta=\log \beta \gamma) .
$$

The coefficients of these equations satisfy three integrability conditions, namely,

$$
\begin{gathered}
\theta_{u v v}=(\gamma \phi)_{u}+2 q_{u}+\theta_{v} \theta_{u v}-\beta \gamma \psi, \\
\theta_{u u v}=(\beta \psi)_{v}+2 p_{v}+\theta_{u} \theta_{u v}-\beta \gamma \phi, \\
p_{v v}-\theta_{v} p_{v}+\beta q_{v}+2 q \beta_{v}=q_{u u}-\theta_{u} q_{u}+\gamma p_{u}+2 p \gamma_{u},
\end{gathered}
$$

where

$$
\phi=\left(\log \beta \gamma^{2}\right)_{u}, \quad \psi=\left(\log \beta^{2} \gamma\right)_{v} .
$$

It is convenient to define $\pi, \chi$ by the formulas

$$
\pi=p+\beta \psi, \quad \chi=q+\gamma \phi .
$$

The most general transformation which leaves Fubini's canonical form invariant is

$$
x=c \bar{x}, \quad \bar{u}=U(u), \quad \bar{v}=V(v) \quad(c=\text { const. }) .
$$

The effect of this transformation on system (1) is to produce another system of the same form whose coefficients, indicated by dashes, are given by the following formulas:

$$
\begin{array}{ll}
\bar{p}=p / U^{\prime 2}, & \bar{\beta}=\beta V^{\prime} / U^{\prime 2} \\
\bar{q}=q / V^{\prime 2}, & \bar{\gamma}=\gamma U^{\prime} / V^{\prime 2}, \quad \bar{\theta}=\theta-\log U^{\prime} V^{\prime} .
\end{array}
$$




\section{Hypergeodesics}

The aim of this section is to present that part of the general theory of hypergeodesics which will be essential for our purposes. Three examples of hypergeodesics will be given.

The two-parameter family of curves defined on an integral surface $S$ of system (1) by a differential equation of the form

$$
v^{\prime \prime}=A+3 B v^{\prime}+3 C v^{\prime 2}+D v^{\prime 3},
$$

where accents indicate total differentiation with respect to $u$, and $A, B, C, D$ are given functions of $u, v$, are called hypergeodesics. These hypergeodesics are covariant to the surface, that is, are geometrically definable in terms of the surface, in case the coefficients in equation (7) are transformed by (5) according to the formulas

$$
\begin{array}{ll}
\bar{A}=A V^{\prime} / U^{\prime 2}, & 3 U^{\prime} \bar{B}=3 B-U^{\prime \prime} / U^{\prime}, \\
\bar{D}=D U^{\prime} / V^{\prime 2}, & 3 V^{\prime} \bar{C}=3 C+V^{\prime \prime} / V^{\prime} .
\end{array}
$$

It will be observed in this connection that $A, D$ are cogredient with $\beta, \gamma$ respectively, while $3 B, 3 C$ are cogredient with $\theta_{u}, \theta_{v}$.

Among the many examples of hypergeodesics that are known, there are three which we shall use. The first example is the curves of a pencil of conjugate nets, that is, a one-parameter family of conjugate nets every one of which has the property that at every point of the surface its tangents form with the tangents of a fundamental conjugate net the same cross ratio. The curvilinear differential equation of a pencil of conjugate nets can be written* in the form

$$
d v^{2}-\lambda^{2} h^{2} d u^{2}=0,
$$

where $\lambda$ is a function of $u, v$, and $h$ is a constant. Eliminating $h$ by differentiation, one obtains an equation of the form (7) with

$$
A=D=0, \quad 3 B=\lambda_{u} / \lambda, \quad 3 C=\lambda_{v} / \lambda .
$$

In order that the pencil may be covariant to the surface it is necessary and sufficient that $\lambda$ be transformed by (5) according to the formula

$$
\bar{\lambda}=\lambda V^{\prime} / U^{\prime} \text {. }
$$

The second example of hypergeodesics is the projective geodesics, which are the extremals of the invariant integral, called the projective arc length,

$$
\int_{u_{1}}^{u_{2}}\left(\beta \gamma v^{\prime}\right)^{1 / 2} d u
$$

\footnotetext{
* Lane, A general theory of conjugate nets, these Transactions, vol. 23 (1922), p. 283.
} 
For these curves one finds, on calculating Euler's equation, a differential equation of the form (7) with

$$
A=D=0, \quad 3 B=\theta_{u}, \quad 3 C=-\theta_{v} .
$$

Finally, the third example is the union curves of a congruence $\Gamma_{1}$. Such a congruence consists of the lines joining each point $x$ of the surface $S$ to the corresponding point

$$
-a x_{u}-b x_{v}+x_{u v},
$$

where $a, b$ are functions of $u, v$. A union curve of this congruence is such that at each of its points its osculating plane contains the line of the congruence through the point. The differential equation of the union curves is of the form (7) with

$$
A=-\beta, \quad 3 B=\theta_{u}-2 b, \quad 3 C=-\theta_{v}+2 a, \quad D=\gamma .
$$

In particular, the union curves of the projective normal congruence are obtained by placing $a=b=0$ in equations (14), and the union curves of the first directrix congruence of Wilczynski by placing therein $2 a=\psi, 2 b=\phi$.

\section{Plane nets}

It is the purpose of this section to summarize the part that we shall need of Wilczynski's theory of plane nets. Then the curvilinear differential equation of the straight lines in the plane will be calculated in Wilczynski's notation, apparently for the first time.

If the three projective homogeneous coördinates $x^{(1)}, x^{(2)}, x^{(3)}$ of a point $P_{x}$ in a plane are given as functions of two parameters $u, v$, these coördinates are solutions of a system of three equations of the form

$$
\begin{aligned}
& x_{u u}=a x_{u}+b x_{v}+c x, \\
& x_{u v}=a^{\prime} x_{u}+b^{\prime} x_{v}+c^{\prime} x, \\
& x_{v v}=a^{\prime \prime} x_{u}+b^{\prime \prime} x_{v}+c^{\prime \prime} x,
\end{aligned}
$$

whose coefficients satisfy* the following six integrability conditions:

$$
\begin{aligned}
a_{v}+a^{\prime \prime} b & =a_{u}^{\prime}+a^{\prime} b^{\prime}+c^{\prime}, \\
b_{u}^{\prime \prime}+a^{\prime \prime} b & =b_{v}^{\prime}+a^{\prime} b^{\prime}+c^{\prime}, \\
b_{v}+a b^{\prime}+b b^{\prime \prime}+c & =b_{u}^{\prime}+a^{\prime} b+b^{\prime 2}, \\
a_{u}^{\prime \prime}+a^{\prime} b^{\prime \prime}+a a^{\prime \prime}+c^{\prime \prime} & =a_{v}^{\prime}+a^{\prime \prime} b^{\prime}+a^{\prime 2}, \\
c_{v}+a c^{\prime}+b c^{\prime \prime} & =c_{u}^{\prime}+a^{\prime} c+b^{\prime} c^{\prime}, \\
c_{u}^{\prime \prime}+b^{\prime \prime} c^{\prime}+a^{\prime \prime} c & =c_{v}^{\prime}+b^{\prime} c^{\prime \prime}+a^{\prime} c^{\prime} .
\end{aligned}
$$

* Wilczynski, One-parameter families and nets of plane curves, these Transactions, vol. 12 (1911), p. 474. 
The effect of the transformation (5) when performed on system (15) is to produce another system of the same form whose coefficients, indicated by dashes, are given by the following formulas:

$$
\begin{aligned}
& U^{\prime} \bar{a}=a-U^{\prime \prime} / U^{\prime}, \quad \bar{b}=b V^{\prime} / U^{\prime 2}, \quad \bar{c}=c / U^{\prime 2}, \\
& \bar{a}^{\prime}=a^{\prime} / V^{\prime}, \quad \bar{b}^{\prime}=b^{\prime} / U^{\prime}, \quad \bar{c}^{\prime}=c^{\prime} / U^{\prime} V^{\prime}, \\
& \bar{a}^{\prime \prime}=a^{\prime \prime} U^{\prime} / V^{\prime 2}, \quad V^{\prime} \bar{b}^{\prime \prime}=b^{\prime \prime}-V^{\prime \prime} / V^{\prime}, \quad \bar{c}^{\prime \prime}=c^{\prime \prime} / V^{\prime 2} \text {. }
\end{aligned}
$$

Moreover, the effect of the transformation

$$
x=\lambda(u, v) \bar{x}
$$

on system (15) is given by the formulas

$$
\begin{array}{rlrl}
\bar{a} & =a-2 \lambda_{u} / \lambda, \quad \bar{b} & =b, \quad \lambda \bar{c}=-\lambda_{u u}+a \lambda_{u}+b \lambda_{v}+c \lambda, \\
\bar{a}^{\prime} & =a^{\prime}-\lambda_{v} / \lambda, \quad \bar{b}^{\prime} & =b^{\prime}-\lambda_{u} / \lambda, \\
\lambda \bar{c}^{\prime} & =-\lambda_{u v}+a^{\prime} \lambda_{u}+b^{\prime} \lambda_{v}+c^{\prime} \lambda, \\
\bar{a}^{\prime \prime} & =a^{\prime \prime}, \quad \bar{b}^{\prime \prime} & =b^{\prime \prime}-2 \lambda_{v} / \lambda \\
\lambda \bar{c}^{\prime \prime} & =-\lambda_{v v}+a^{\prime \prime} \lambda_{u}+b^{\prime \prime} \lambda_{v}+c^{\prime \prime} \lambda .
\end{array}
$$

The differential equation of the straight lines of the plane can be calculated by making use of the fact that $x, x^{\prime}, x^{\prime \prime}$ must satisfy a linear relation when the accents indicate total differentiation with respect to $u$ along a straight line of the plane. We have at once

$$
\begin{aligned}
x^{\prime} & =x_{u}+x_{v} v^{\prime}, \\
x^{\prime \prime} & =x_{u u}+2 x_{u v} v^{\prime}+x_{v v} v^{\prime 2}+x_{v} v^{\prime \prime} .
\end{aligned}
$$

Substituting for the second derivatives in the second of these equations the expressions given for them in equations (15), we can express $x^{\prime \prime}$ as a linear combination of $x, x_{u}, x_{v}$. Regarding each of $x, x^{\prime}, x^{\prime \prime}$ as so expressed, and equating to zero the third-order determinant of the coefficients of $x$, $x_{u}, x_{v}$ in their expressions, we obtain the curvilinear differential equation of the straight lines in the plane, namely,

$$
v^{\prime \prime}=-b+\left(a-2 b^{\prime}\right) v^{\prime}-\left(b^{\prime \prime}-2 a^{\prime}\right) v^{\prime 2}+a^{\prime \prime} v^{\prime 3} .
$$

\section{HYPERGEODESIC MAPPING}

In this section we solve the problem of mapping a surface, as defined in $\S 2$, on a plane so that the covariant hypergeodesics of $\$ 3$ correspond to the straight lines in the plane. The coefficients of the differential equations of the plane net corresponding to the asymptotic curves on the surface are determined in terms of the coefficients in the equation of the hypergeodesics, 
and their derivatives. It is found that these coefficients must satisfy two conditions in order that the mapping in question may be possible.

Let us suppose that we have a one-to-one correspondence between an integral surface $S$ of system (1) and an integral plane net $N$ of equations (15), or at least between certain regions of these, such that corresponding points have the same curvilinear coördinates $u, v$. Moreover, let us demand that a given family of covariant hypergeodesics (7) shall correspond to the straight lines (21). Conditions necessary and sufficient therefore are

$$
\begin{aligned}
b=-A, \quad a-2 b^{\prime} & =3 B, \\
b^{\prime \prime}-2 a^{\prime} & =-3 C, \quad a^{\prime \prime}=D .
\end{aligned}
$$

It may be remarked that the left members of these equations are shown by the formulas (19) to be absolutely invariant under the transformation (18), as they should be. Moreover, these equations are shown by equations (8) and (17) to be absolutely invariant under the transformation (5).

The net $N$ is the map of the asymptotic curves on the surface $S$. If $A=D=0$, the asymptotic curves on $S$ belong to the given family of hypergeodesics, and map into two one-parameter families of straight lines composing the net $N$.

Let us attempt to calculate the coefficients of system (15) in terms of $A, B, C, D$ and the derivatives of these functions. To this end, let us solve equations (22) for $a, b, a^{\prime \prime}, b^{\prime \prime}$, and substitute the resulting expressions for these four coefficients in the integrability conditions (16), obtaining

$$
\begin{aligned}
\left(2 b^{\prime}+3 B\right)_{v}-A D & =a_{u}^{\prime}+a^{\prime} b^{\prime}+c^{\prime}, \\
\left(2 a^{\prime}-3 C\right)_{u}-A D & =b_{v}^{\prime}+a^{\prime} b^{\prime}+c^{\prime}, \\
-A_{v}+b^{\prime}\left(b^{\prime}+3 B\right)-A\left(a^{\prime}-3 C\right)+c & =b_{u}^{\prime} \\
D_{u}+a^{\prime}\left(a^{\prime}-3 C\right)+D\left(b^{\prime}+3 B\right)+c^{\prime \prime} & =a_{v}^{\prime} \\
c_{v}+c^{\prime}\left(b^{\prime}+3 B\right)-A c^{\prime \prime} & =c_{u}^{\prime}+a^{\prime} c, \\
c_{u}^{\prime \prime}+c^{\prime}\left(a^{\prime}-3 C\right)+D c & =c_{v}^{\prime}+b^{\prime} c^{\prime \prime} .
\end{aligned}
$$

The first two of these equations imply

$$
\left(b^{\prime}+B\right)_{v}=\left(a^{\prime}-C\right)_{u} .
$$

Consequently, equations (19) show that if $\lambda$ is a solution of the equations

$$
(\log \lambda)_{u}=b^{\prime}+B, \quad(\log \lambda)_{v}=a^{\prime}-C,
$$

then, dropping dashes from the transformed coefficients, we shall have

$$
a^{\prime}=C, \quad \quad b^{\prime}=-B .
$$

The first, third, and fourth of equations (23) now give expressions for $c^{\prime}$, $c, c^{\prime \prime}$ respectively, while the last two of equations (23) give two conditions 
that must be satisfied by $A, B, C, D$. We reach thus the following conclusion.

The hypergeodesics (7) on an integral surface $S$ of system (1) can be mapped into the straight lines of the plane if, and only if, the coefficients $A, B, C, D$ in equation (7) satisfy the two conditions

$$
\begin{aligned}
2 B_{u v}-A_{v v}-C_{u u}-6 B B_{v}+3 A C_{v}-A_{u} D-2 A D_{u}+3 B C_{u}+3 C A_{v} & =0 \\
-2 C_{u v}+D_{u u}+B_{v v}-6 C C_{u}+3 D B_{u}-D_{v} A-2 D A_{v}+3 C B_{v}+3 B D_{u} & =0 .
\end{aligned}
$$

The parametric asymptotic curves on the surface $S$ map into an integral net $N$ of the system (15) whose coefficients are given by the formulas

$$
\begin{aligned}
a & =B, \quad b & =-A, \quad c & =A_{v}-B_{u}+2 B^{2}-2 A C, \\
a^{\prime} & =C, \quad b^{\prime} & =-B, \quad c^{\prime} & =B_{v}-C_{u}-A D-B C, \\
a^{\prime \prime} & =D, \quad b^{\prime \prime} & =-C, \quad c^{\prime \prime} & =-D_{u}+C_{v}+2 C^{2}-2 B D .
\end{aligned}
$$

The integrability conditions of this system (15) are all satisfied.

\section{EXAMPLES OF HYPERGEODESIC MAPPING}

Three examples of hypergeodesic mapping, corresponding to the three special kinds of hypergeodesics mentioned in $\$ 3$, will now be discussed.

For the first example, let us consider the curves of a pencil of conjugate nets. The conditions (27) reduce, in virtue of equations (10), to

$$
\mu_{u u v}-\mu_{u} \mu_{u v}=0, \quad \mu_{u v v}-\mu_{v} \mu_{u v}=0,
$$

where $\mu$ is defined by placing

$$
\mu=\log \lambda \text {. }
$$

Integrating each of equations (29), one obtains

$$
\mu_{u u}-\frac{1}{2} \mu_{u}^{2}=U_{1}, \quad \mu_{v v}-\frac{1}{2} \mu_{v}^{2}=V_{1},
$$

where $U_{1}, V_{1}$ are arbitrary functions of $u$ alone and of $v$ alone, respectively. By means of equation (11) it is possible to show that a transformation (5) for which we have

$$
\begin{aligned}
\mu_{u u}-\frac{1}{2} \mu_{u}{ }^{2} & =U^{\prime \prime \prime} / U^{\prime}-3 U^{\prime \prime 2} / 2 U^{\prime 2}, \\
\mu_{v v}-\frac{1}{2} \mu_{v}{ }^{2} & =V^{\prime \prime \prime} / V^{\prime}-3 V^{\prime \prime 2} / 2 V^{\prime 2},
\end{aligned}
$$

reduces each of $U_{1}$ and $V_{1}$ to zero. Incidentally, the most general transformation (5) which leaves these conditions undisturbed has $U$ and $V$ equal to proper linear fractional functions with constant coefficients so that

$$
U=(a u+b) /(c u+d), \quad V=\left(a^{\prime} v+b^{\prime}\right) /\left(c^{\prime} v+d^{\prime}\right) .
$$

We now have

$$
\mu_{u u}+\frac{1}{2} \mu_{u}^{2}=0, \quad \mu_{v v}-\frac{1}{2} \mu_{v}^{2}=0 .
$$


Integration of these equations leads to the formula for $\lambda$

$$
\lambda=1 /(k u v+l u+m v+n)^{2},
$$

in which $k, l, m, n$ are constants. Therefore, the curves of a pencil of conjugate nets (9) with $\lambda$ given by the formula (35) map into the straight lines of the plane.

Two remarks concerning this result may be permitted. A pencil of the kind described is a pencil of isothermally conjugate nets, characterized by the condition $(\log \lambda)_{u v}=0$, if, and only if, $k=0$. If $k \neq 0$, it is possible, by means of a suitably chosen transformation (33), to make $l=m=0$.

For the second example of hypergeodesic mapping, let us consider the projective geodesics. The conditions (27) reduce, in virtue of equations (13), to

$$
\theta_{u u v}-\theta_{u} \theta_{u v}=0, \quad \theta_{u v v}-\theta_{v} \theta_{u v}=0 .
$$

Proceeding step by step as in the preceding example, we find that the projective geodesics on an integral surface of system (1) map into the straight lines of the plane if, and only if,

$$
\beta \gamma=1 /(k u v+l u+m v+n)^{2},
$$

where $k, l, m, n$ are constants. If $k \neq 0$, we can make $l=m=0$.

For the third example of hypergeodesic mapping, let us consider the union curves of the projective normal congruence. We shall not write here the result of substituting in the conditions (27) the general expressions given for $A, B, C, D$ in equations (14). However, when $a=b=0$, this substitution leads to

$$
\begin{aligned}
& \theta_{u u v}-\theta_{u} \theta_{u v}+(\beta \psi)_{v}+\beta \gamma \phi=0, \\
& \theta_{u v v}-\theta_{v} \theta_{u v}+(\gamma \phi)_{u}+\beta \gamma \psi=0,
\end{aligned}
$$

where $\phi, \psi$ are defined in equations (3). By means of the first two integrability conditions (2), one obtains at once

$$
(p+\beta \psi)_{v}=0, \quad(q+\gamma \phi)_{u}=0
$$

and on integrating these equations one arrives at

$$
p+\beta \psi=U_{1}, \quad q+\gamma \phi=V_{1} .
$$

By means of a suitably chosen transformation (5) it is possible to reduce each of $U_{1}$ and $V_{1}$ to unity, so that $\pi=\chi=1$, the functions $\pi, \chi$ being defined in equations (3). Therefore, the union curves of the projective normal congruence of an integral surface of a system (1) for which

$$
p=1-\beta \psi, \quad q=1-\gamma \phi,
$$


map into the straight lines of the plane.

A consequence of this result may be deduced. The differential equation of the projective lines of curvature, which are the curves corresponding to the developables of the projective normal congruence, is

$$
\pi d u^{2}-\chi d v^{2}=0 .
$$

Therefore, on a surface of the kind under consideration here the projective lines of curvature form an isothermally conjugate net, since $\pi=\chi=1$.

\section{The CONGRUENTIALly ASSOciated NET}

The congruentially associated net of a given plane net was defined* by Green as follows. As a point $P_{x}$ varies along a curve of the congruentially associated net, the harmonic conjugate of the tangent of this curve, with respect to the two tangents of the given net, passes through the corresponding focal point of the ray of the given net. The curvilinear differential equation of the congruentially associated net of an integral net of system (15) is

$$
\begin{aligned}
{\left[c+b^{\prime}\left(a-b^{\prime}\right)+a^{\prime} b-b_{u}^{\prime}\right] } & d u^{2}+\left(a_{u}^{\prime}-b_{v}^{\prime}\right) d u d v \\
- & {\left[c^{\prime \prime}+a^{\prime}\left(b^{\prime \prime}-a^{\prime}\right)+a^{\prime \prime} b^{\prime}-a_{v}^{\prime}\right] d v^{2}=0 . }
\end{aligned}
$$

By means of equations (28) this equation reduces to

$$
\left(A_{v}-3 A C\right) d u^{2}+\left(B_{v}+C_{u}\right) d u d v+\left(D_{u}+3 B D\right) d v^{2}=0 .
$$

This is the differential equation of the congruentially associated net of the $n e t N$ into which the asymptotics on the surface $S$ map when the hypergeodesics on $S$ map into the straight lines in the plane of the net $N$.

It is easy to prove the following theorems. In the case of the curves of a pencil of conjugate nets the congruentially associated net coincides with the net $N$, provided that the nets of the pencil of conjugate nets are not isothermally conjugate. If they are isothermally conjugate, the congruentially associated net is indeterminate. In the case of the projective geodesics the congruentially associated net is indeterminate.

When the union curves of a congruence $\Gamma_{1}$ are mapped into the straight lines in a plane, the congruentially associated net of the net $N$ has the differential equation

$$
\beta(\psi-2 a) d u^{2}+2\left(b_{v}-a_{u}\right) d u d v / 3-\gamma(\phi-2 b) d v^{2}=0 .
$$

The coefficients of $d u^{2}$ and $d v^{2}$ vanish if the congruence $\Gamma_{1}$ is the first directrix congruence of Wilczynski. In this case the conditions (27) reduce to

\footnotetext{
* Green, Plane nets with equal invariants, Annals of Mathematics, vol. 19 (1918), p. 248.
} 


$$
\begin{aligned}
& \phi_{u v}+\phi_{v}(\log \gamma)_{u}-3 \beta \gamma \phi=0, \\
& \psi_{u v}+\psi_{u}(\log \beta)_{v}-3 \beta \gamma \psi=0 .
\end{aligned}
$$

We reach thus the following theorem.

If the surface $S$ is not isothermally asymptotic, so that $\phi_{v}-\psi_{u} \neq 0$, the first directrix congruence of Wilczynski is the only congruence $\Gamma_{1}$ whose union curves map into the straight lines of a plane so that the congruentially associated net of the net $N$ corresponding to the asymptotics coincides with the net $N$. If the surface $S$ is isothermally asymptotic, the congruentially associated net of the net $N$ is indeterminate in the case of the union curves of the directrix congruence.

Placing $a=b=0$ in equation (45) to obtain the differential equation of the congruentially associated net in the case of the union curves of the projective normal congruence, one sees that, in this case, provided $\phi \psi \neq 0$, at each point of the plane the tangents of the congruentially associated net of the net $N$, corresponding to the asymptotics on the surface $S$, separate harmonically the tangents of the net $N$. The cases $\phi \psi=0$ are easily disposed of.

\section{ANOTHER METHOD OF MAPPING}

It may be proposed to map a surface onto a plane so that the hypergeodesics on the surface correspond to any two-parameter family of curves defined in the plane by a differential equation of the same form as that of the hypergeodesics. So far as the writer is aware, the projective differential aspects of such families of curves in the plane have not hitherto been studied. It would seem, therefore, that the following example of such a family of curves covariant to a given plane net may be of interest.

Equations (17), (19) show that the differential form $a^{\prime \prime} b d u d v$ is absolutely invariant under the most general transformation leaving invariant an integral net of system (15). Therefore the integral

$$
\int_{u_{0}}^{u}\left(a^{\prime \prime} b v^{\prime}\right)^{1 / 2} d u
$$

has a geometric significance. Euler's equation for the extremals of this integral is of the same form as equation (7), with

$$
A=D=0, \quad 3 B=\left(\log a^{\prime \prime} b\right)_{u}, \quad 3 C=-\left(\log a^{\prime \prime} b\right)_{v} .
$$

These extremals constitute a family of curves covariant to the integral net of system (15).

The projective geodesics on an integral surface $S$ of system (1) map into these curves in case

$$
(\log \beta \gamma)_{u}=\left(\log a^{\prime \prime} b\right)_{u}, \quad(\log \beta \gamma)_{v}=\left(\log a^{\prime \prime} b\right)_{v} .
$$


Integrating, and absorbing a constant factor into the product $a^{\prime \prime} b$, we see that the projective geodesics on the surface $S$ map into the plane curves given by equation (46) and covariant to the plane net $N$ corresponding to the asymptotic curves on $S$ if, and only if,

$$
a^{\prime \prime} b=\beta \gamma .
$$

It may be remarked that if the plane net $N$ is perspective to the asymptotic net on the surface $S$, then the center of perspectivity and the plane of the net $N$ may be chosen to be the point $(0,0,0,1)$ and the plane $x_{4}=0$. Then it follows that $\beta=b, \gamma=a^{\prime \prime}$, so that the perspectivity is a mapping of the kind under consideration here.

It is clear that the bundle of planes with center at the center of perspectivity is a family of $\infty^{2}$ planes cutting the surface $S$ in $\infty^{2}$ plane curves which are union curves of the congruence $\Gamma_{1}$ which is the bundle of lines with its center at the center of perspectivity. Moreover, these planes cut the plane of the net $N$ in the straight lines of this plane. Therefore we have here an instance of the mapping of the union curves of a congruence $\Gamma_{1}$ into the straight lines of the plane. It may be shown, moreover, that if a family of hypergeodesics on the surface $S$ corresponds to the straight lines of the plane in the perspectivity aforesaid, then the hypergeodesics are the union curves of the bundle of lines with center at the center of perspectivity, and are precisely the plane curves just described on the surface $S$.

University of Chicago, Chicago, Ill. 